\title{
Measuring Strain in AIN/GaN Superlattices and Nanowires by NanoBeam Electron Diffraction
}

\author{
C. Bougerol*, A. Béché**, B. Daudin***, J.L. Rouvière*** \\ * Institut Néel, CNRS, BP 166, 25 rue des Martyrs, 38042 Grenoble Cedex 9, France \\ ** FEI Company, Achtseweg Noord 5, 5651 GG Eindhoven, The Netherlands \\ *** CEA-Grenoble, INAC/SP2M, 38054 Grenoble, France
}

Nanobeam electron diffraction (NBED or NBD) is a quite recent transmission electron microscopy (TEM) based technique [1] able to measure strain at the nanoscale with a spatial resolution down to $3 \mathrm{~nm}$ and a strain sensitivity of $6.10^{-4}$ [2]. It consists in illuminating a sample with an almost parallel electron probe and recording the resulting diffraction pattern. Monitoring the position of the diffraction spots in the diffraction plane gives access to the strain in the two directions orthogonal to the electron beam. The specificity of NBED lays in the fact that the reference region does not need to be close to the region of interest contrary to high resolution TEM [3] or the more recent dark field electron holography technique [4]. In the most critical cases, the reference diffraction pattern can even be acquired on another sample. This ability allows measuring strain even in very small devices like nanowires as we show here.

In a first set of experiments, short periods $\mathrm{AlN}-\mathrm{GaN}$ superlattices deposited on $\mathrm{SiC}$ substrate were studied (Fig. 1.a). The probe diameter was set to $4 \mathrm{~nm}$ (Fig. 1.b) with a convergence angle of 0.4 mrad (Fig. 1.c). These measurements allowed checking for the quantitative strain measurement of these materials as it is easy to model them with finite element method (Fig. 1.d). Moreover, it gives the occasion to evaluate the influence of convolution on the probe size relative to the strained layer thickness.

In a second set of experiments, AlN/GaN nanowires of $45 \mathrm{~nm}$ in diameter were studied. On top of $\mathrm{GaN}$ nanowires, AlN and GaN layers were alternatively deposited leading to $3 \mathrm{~nm}$ thick GaN insertions that are separated by AlN barriers and that optically behaves as quantum dots (Fig. 2.a). To achieve quantitative measurement on such small devices, probe size was reduced to $2 \mathrm{~nm}$ in diameter in detriment to convergence angle. We have been able to follow quantitatively the strain along the growth axis (Fig. 2.b). These results should allow to better understand the role of the strain on the luminescence properties of such AlN/GaN nanostructures.

References

[1] K. Usuda et al., Applied Surface Science 224, 1132004.

[2] A. Béché, J. Rouvière, L. Clément, J. Hartmann, Applied Physics Letters 95, 123114, 2009.

[3] Sarigiannidou, E. et al., Applied Physics Letters 87, 203112-3, 2005.

[4] M. Hÿtch, F. Houdellier, F. Hue, E. Snoeck, Nature 453, 1086 (2008). 

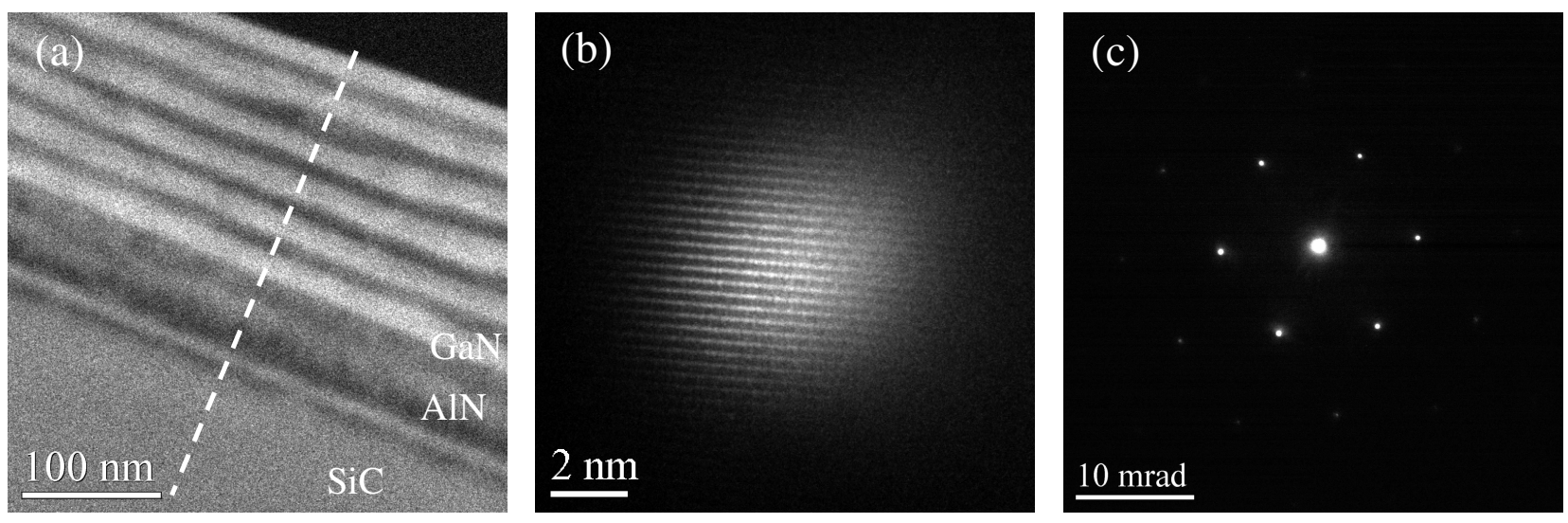

(d)

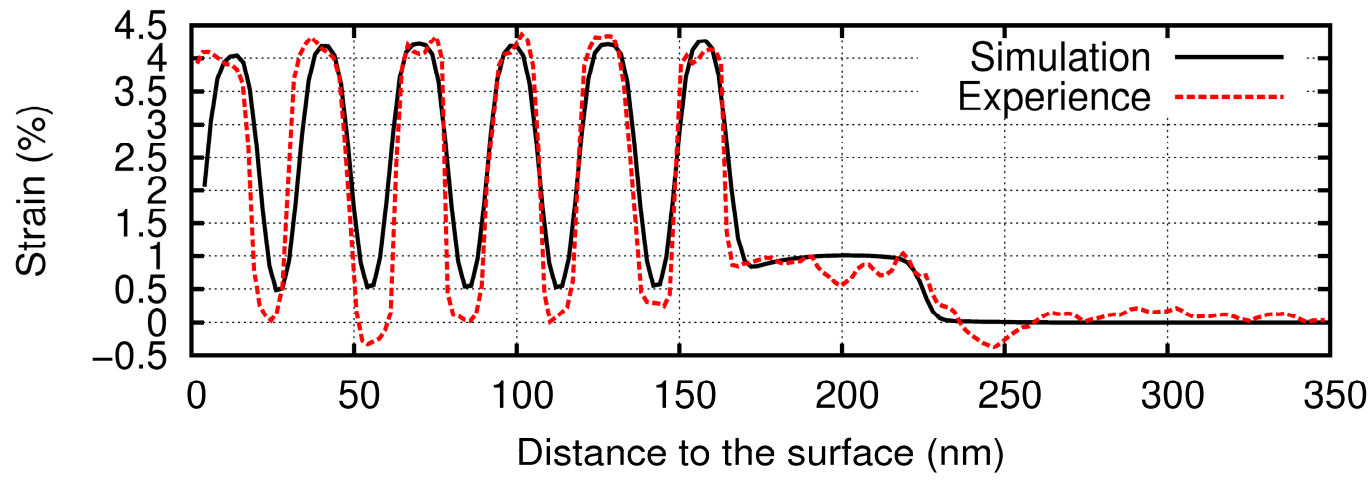

FIG. 1. (c) Image of the sample using the NBED probe (b). (b) Image of the NBED electron probe through the sample. (c) Diffraction pattern corresponding to the probe in (b). (d) Experimental strain profile in the growth direction compared to simulation.
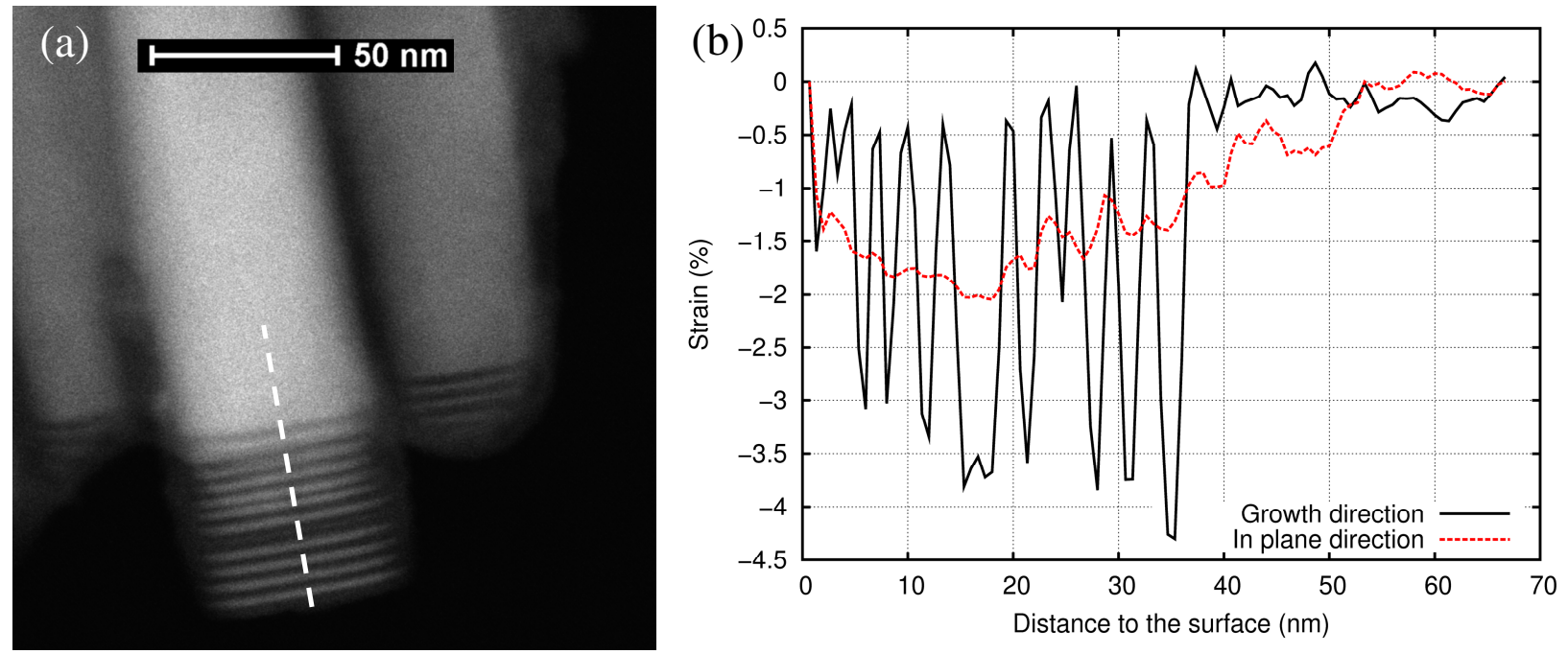

FIG. 2. (a) AlN-GaN nanowires with $3 \mathrm{~nm}$ thick quantum dots. (b) Strain profiles of nanowires. 